Revue Interventions économiques

Papers in Political Economy

Hors-série. Transformations | 2017

L'Afrique est-elle partie? Bilan et perspectives de l'intégration africaine

\title{
Le rôle et la place des communautés économiques régionales dans le développement de l'Afrique
}

The Role and Place of Regional Economic Communities in Africa's Development

\section{Léonard Matala-Tala}

\section{OpenEdition}

\section{Journals}

Édition électronique

URL : https://journals.openedition.org/interventionseconomiques/5699

DOI : 10.4000/interventionseconomiques.5699

ISSN : $1710-7377$

Éditeur

Association d'Économie Politique

Référence électronique

Léonard Matala-Tala, "Le rôle et la place des communautés économiques régionales dans le développement de l'Afrique », Revue Interventions économiques [En ligne], Hors-série. Transformations | 2017, mis en ligne le 01 mars 2017, consulté le 21 mars 2023. URL : http://journals.openedition.org/ interventionseconomiques/5699; DOI : https://doi.org/10.4000/interventionseconomiques.5699

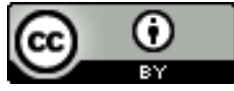

Creative Commons - Attribution 4.0 International - CC BY 4.0

https://creativecommons.org/licenses/by/4.0/ 
Par une sorte de mimétisme

institutionnel, les africains veulent

obtenir en 27 ans, ce que les

Européens ont construit

progressivement sur plus de 60 ans.

A travers l'Agenda 2063, les dirigeants africains ont exprimé une 'vision panafricaine' du futur: " une Afrique intégrée, prospère et pacifique, dirigée par ses propres citoyens et représentant une force dynamique sur la scène internationale $»$.

A l'origine, la plupart de mécanismes transfrontaliers coloniaux ont été maintenus après les indépendances africaines afin d'appuyer le programme d'intégration régionale. C'est ainsi que l'ancienne Communauté financière africaine (CFA) avec son Franc a été intégrée à la CEDEAO et à la CEMAC. En 1994, le traité d'Abuja appelle à instituer une Communauté économique africaine en jetant les bases d'une intégration africaine dont les Communautés économiques régionales (CERs) sont les principaux vecteurs. De nombreux arguments plaident pour le renforcement de l'intégration régionale africaine : faiblesse des échanges internes, perméabilité des frontières, vétusté ou absence des infrastructures. Cette dimension économique fait partie d'un dispositif qui justifie les aides des bailleurs de fonds tandis que la mondialisation relance la question des 'territoires pertinents' pour les échanges mondiaux.

On distingue quatorze grands groupements économiques régionaux (Gbaguidi 2013 : 50) plus ou moins intégrés. Cette étude évoque essentiellement les CERs suivantes:

\begin{tabular}{|c|c|c|c|}
\hline Dénomination & $\begin{array}{l}\text { Année de } \\
\text { création }\end{array}$ & Siège & Nombre dÉtats membres \\
\hline $\begin{array}{c}\text { UNA } \\
\text { (Union du Maghreb arabe) }\end{array}$ & 1989 & Rabat & 5 \\
\hline $\begin{array}{c}\text { CEDEAO } \\
\text { (Communauté économique des États de } \\
\text { lAfrique de lOuest) }\end{array}$ & 1976 & Lomé & 15 \\
\hline $\begin{array}{c}\text { EAC } \\
\text { (East African community) }\end{array}$ & 1999 & Arusha & 6 \\
\hline $\begin{array}{c}\text { IGAD } \\
\begin{array}{c}\text { (Intergovernmental Authority on } \\
\text { development) }\end{array}\end{array}$ & 1986 & Djibouti & 7 \\
\hline $\begin{array}{c}\text { CEMAC } \\
\text { (Communauté économique et monétaire de } \\
\text { 1Afrique centrale) }\end{array}$ & 1999 & Libreville & 11 \\
\hline $\begin{array}{l}\text { COMISA } \\
\text { (Commonmarket for eastem et southern } \\
\text { Africa) }\end{array}$ & 1994 & Lusaka & 19 \\
\hline $\begin{array}{c}\text { SADC } \\
\text { (SouthernAfrican development community) }\end{array}$ & 1980 & Gaborone & 14 \\
\hline
\end{tabular}

Le rôle des CERs leur permet-il d'être réellement les " pierres angulaires de l'unité du continent " (Union africaine, $2015: 1)^{2}$ ? Quels sont les atouts et les freins de l'intégration régionale (CER) dans sa contribution au développement de l'Afrique? Ces CERs prennent-elles part au partenariat Afrique-Union européenne? Cette analyse conduit au constat selon lequel, malgré une existence juridique incontestable, ces CERs ont des moyens très limités (I), et sont encore en quête d'un rôle effectif (II).

\section{Une existence juridique incontestable mais des moyens limités}

Sur les 54 pays africains (Gbaguidi $2013: 51$ ), 27 sont membres de 2 groupements régionaux, 18 appartiennent à 3 groupements, 1 pays est membre de 4 groupements tandis que 8 pays seulement ne sont membres que d'un seul groupement. Toutes les CERs africaines poursuivent les mêmes objectifs : intégration économique, libre circulation, établissement d'une union douanière précédant une union économique et monétaire et une union politique, le tout sur fond de paix et sécurité. Elles ont toutes des structures institutionnelles multiples avec un grand écart (Vanheukelom et al., 2016) entre leurs objectifs et les fonctions qu'elles remplissent réellement.

Par une sorte de mimétisme institutionnel, les africains veulent obtenir en 27 ans, ce que les Européens ont construit progressivement sur plus de 60 ans - de la CECA en 1951, puis la CEE en 1957, pour atteindre en 1992, une Union européenne encore inachevée aujourd'hui. Sauf que du côté africain, on semble avoir négligé un préalable fondamental à toute intégration: la volonté politique et l'adoption de valeurs communes ${ }^{3}$. Dès 1951, les États européens, certes traumatisés par la guerre, partageaient néanmoins un certain nombre de valeurs ${ }^{4}$ : liberté, démocratie, égalité, État de droit, respect des droits de l'homme, pluralisme, non-discrimination, tolérance, justice, solidarité et 
égalité hommes femmes. Ils ont ainsi adopté une législation et un tarif douanier communs et construit les infrastructures nécessaires à l'édification d'un espace sans frontières intérieures dans lequel les marchandises, services, capitaux et personnes circulent librement.

Les Éłats africains sont loin de ce degré de convergence tant leurs situations politiques et économiques sont hétérogènes. Les CERs pourraient être les pierres angulaires de cette convergence, encore faut-il que les dirigeants expriment une réelle volonté politique et adoptent des valeurs africaines communes. Le fonctionnement voire la survie des CERs est fortement tributaire des États membres. En effet, tant que «les dirigeants nationaux conservent la main haute dans les instances de décision, les CERs restent très timides lorsqu'il s'agit de dénoncer des cas de fraude électorale ou de répression policière organisée par les États $11^{5}$. Pour sortir de cette impasse, des États locomotives devraient émerger au sein de chaque CER, à l'image du couple franco-allemand, pour faire avancer l'intégration régionale. Pour le moment, la situation politique des États comme l'Algérie, le Nigeria, la République démocratique du Congo, l'Angola et la République Sud africaine, qui pourraient jouer ce rôle, fragilisent le processus. C'est là l'un des handicaps qui affectent ces organisations qui se cherchent encore.

Pour harmoniser les législations et les pratiques, la création d'un mécanisme officiel de suivi permettrait « d'identifier, de manière rationnelle et objective, les pays réalisant le plus de progrès en matière d'intégration régionale, les domaines dans lesquels certains pays prennent du retard, et les politiques et institutions qui s'avèrent les plus efficaces dans leurs efforts de promotion de l'intégration régionale " (Davis, 2016). En outre, les États devraient habiliter leurs CERs à disposer des ressources propres pour remplir leurs missions. Sans tout cela, il sera difficile d'atteindre les objectifs du traité d'Abuja.

\section{Une quête continue et ardue d'un rôle effectif sur le terrain}

Les initiatives pour renforcer l'intégration africaine ne manquent pas : zone de libre-échange continentale $^{6}$, zone de libre-échange tripartite ${ }^{7}$, ou tarif extérieur commun en Afrique de l'Ouest. Les CERs devraient conduire l'Afrique à abandonner son statut de fournisseur de produits de base (Dieye, 2015) et de matières premières qui la confine actuellement au bas des chaînes des valeurs globales.

Mais sur le terrain, les CERs sont loin d'avoir atteint leurs objectifs de développement régional et leurs performances sont variables : «seules la CAE et la CEDEAO sont [...] qualifiées de zones en voie de développement régional " (Gbaguidi, 2013 : 54). Elles sont en avance par rapport aux autres CERs en matière de libre circulation des personnes car elles délivrent déjà un passeport commun. Toutes les CERs, exceptée la CEDEAO, doivent aussi leur survie à une subvention extérieure (Cour des comptes européennes, 2010), ce qui est une grande faiblesse congénitale. Sur le plan économique, le commerce intra-africain reste très faible (Gbaguidi, 2013 : 55) avec environ 4\% pour l'UMA, 9,2\% pour la CEDEAO, ou $9,8 \%$ pour la SADC, inférieur à $1 \%$ au niveau continentale ${ }^{8}$, alors que ce taux est d'environ $19 \%$ pour le MERCOSUR, $21 \%$ pour l'ASEAN, et $60 \%$ pour I'Union européenne. Un des obstacles dans ce domaine est "qu'aucune des monnaies nationales, ou presque, n'est convertible, à l'exception du Franc CFA ॥ (Gbaguidi, 2013 : 57). Par ailleurs, l'Afrique pourrait fonder sa relance sur les services (UNCTAD/ALDC/AFRICA, 2015) et l'énergie. La percée des TIC a fortement fait progresser l'interconnectivité des États, et ipso facto, l'accroissement des échanges et la privatisation des services nationaux. La difficulté est que de nombreux réseaux routiers, aériens et ferroviaires sur l'ensemble du continent ne sont toujours pas interconnectés et certaines CERs (SADC, CEDEAO, COMESA, UMA) le sont mieux que d'autres. La pratique des échanges transfrontaliers informels peut servir de trame pour organiser et affermir cette dynamique intégratrice.

Ce constat n'est pas à la hauteur des potentialités africaines (humaines, naturelles et technologiques) immenses mais sous exploitées et entravées. La mise en œuvre de projets ambitieux, bute sur l'instabilité politique et la pauvreté qui règnent dans la plupart des pays africains. Ainsi, presque tous les États membres de la CEMAC ont connu des soubresauts politiques dans les deux années écoulées: Burundi, Congo, Gabon, Guinée-Bissau, RCA, RDC, Rwanda et Tchad. On déplore donc toujours un manque de soutien effectif à la paix, à la stabilité et à la

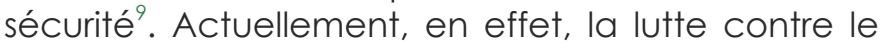
terrorisme, le fanatisme religieux, la piraterie, les trafics des stupéfiants et d'êtres humains qui affectent dangereusement la souveraineté et l'intégrité territoriale des États africains, se fait la plupart du temps grace à une intervention extérieure (Mali, RCA, Tunisie, etc.).

L'Union africaine et les CERs devraient « prendre des mesures fortes pour restaurer et maintenir la paix et la stabilité dans les régions touchées par des conflits " (UNECA/OCDE, 2015 : 7). Dans cette perspective, elles méritent un soutien ferme des États africains. Elles devraient aussi prendre les devants dans les négociations internationales. L'article 6 de l'Accord de Cotonou reconnaît aux CERs le rôle d'acteur. A ce titre, elles reçoivent une assistance technique et 
financière de l'Union européenne notamment pour le renforcement de leurs capacités institutionnelles, la création de marchés régionaux intégrés, le soutien au secteur privé et le raccordement des réseaux d'infrastructures entre eux. Toutefois, il importe d'impliquer davantage les CERs dans ce partenariat entre l'Afrique et l'Union européenne, car elles ont actuellement un rôle marginal.

Une vraie intégration régionale exige changement des mentalités, pragmatisme, rythme et cohérence, pertinence et compatibilité des priorités. Les États et les CERs doivent démontrer une réelle volonté d'atteindre cet objectif, en ciblant les besoins, clarifiant les missions, repensant leur mise en œuvre, en s'appuyant sur les atouts existants, pour permettre le développement et assurer sa pérennité. Enfin, les CERs doivent rendre compte de leurs actions.

1 Commission des Nations unies pour l'Afrique, Historique des efforts déployés en Afrique en appui à l'intégration régionale, visible sur http://www.uneca.org/fr/oria/pages/historique-des-efforts-d\%C3\%A9ploy\%C3\%A9s-en-afrique-en-appui-\%C3\%A0-1\%E2 \%80\%99int\%C3\%A9gration-r\%C3\%A9gionale, consultée le 16 janvier 2017.

2 Union africaine, Agenda 2063, l'Afrique que nous voulons, troisième édition, Addis Ababa, janvier 2015, point 3, p.1.

3 Terence Corrigan, African unity will remain illusionary without values, disponible sur http://www.saiia.org.za/opinion-analysis/african-unity-will-remain-illusionary-without-values; consultée le 16 janvier 2017 in SAllA Research Report, Building Freedom? Securing Constitutionalism and Civil Liberties in Africa - An Analysis of Evidence from the APRM.

4 Article 2 du Traité sur l'Union européenne, version consolidée, Journal officiel de l'Union européenne, C326, 26 octobre 2012, p.17.

5 Vincent ROUGET, Quel bilan pour l'intégration régionale en Afrique ? in http://terangaweb.com/quel-bilan-lintegration-regionale-en-afrique/ consultée le 16 janvier 2017.

6 Union africaine, feuille de route pour une mise en place en 2017 d'une Continental Free Trade Area. http://www.au.int/en/ti/cfta/about consultée le 16 janvier 2017.

7 Troisième sommet tripartite tenu à Charm el-Cheikh le 10 juin 2015, pour constituer la zone de libre-échange tripartite (Tripartite Free Trade Area - TFTA) de 26 Etats.

8 Vincent ROUGET, Quel bilan pour l'intégration régionale en Afrique? disponible sur http://terangaweb.com/quel-bilan-lintegration-regionale-en-afrique/ consultée le 16 janvier 2017.

9 II faut néanmoins saluer la CEDEAO qui dispose d'une force armée (ex-ECOMOG) et qui vient de mettre un terme à la crise politique gambienne de décembre 2016, mais qui reste inefficace face à Boko haram et Ansaru au Nigeria.

\section{Références bibliographiques}

Commission économique des Nations unies pour l'Afrique (Page consultée le 16 janvier 2017). Historique des efforts déployés en Afrique en appui à l'intégration régionale, visible sur http://www.uneca.org/fr/oria/pages/historique-des-efforts-d\%C3\%A9ploy\%C3\%A9s-en-afrique-en-appui-\%C3\%A0-1\%E2 \%80\%99int\%C3\%A9gration-r\%C3\%A9gionale.

Commission économique des Nations unies pour l'Afrique et Organisation de coopération pour le développement économique, Examen mutuel de l'efficacité du développement en Afrique : promesses \& résultats. Rapport conjoint, 2015.

Conférence des Nations unies sur le commerce et le développement (2015). Le développement économique en Afrique Rapport 2015, Libérer le potentiel du commerce des services en Afrique pour la croissance et le développement, UNCTAD/ALDC/AFRICA/2015.

Corrigan, T., African unity will remain illusionary without values, disponible sur http://www.saiia.org.za/opinion-analysis/african-unity-will-remain-illusionary-without-values; consultée le 16 janvier 2017 in SAllA Research Report, Building Freedom? Securing Constitutionalism and Civil Liberties in Africa - An Analysis of Evidence from the APRM.

Cour des comptes européenne (2010). Rapport spécial n¹8/2009. Efficacité de l'appui du FEd à l'intégration économique régionale en Afrique de l'Est et de l'Ouest.

Davis, W. (Page consultée le 16 janvier 2017). Assurer le suivi de l'intégration régionale en Afrique, Passerelles, Vol.17 n8, 26 October 2016, http://www.ictsd.org/bridges-news/$\mathrm{p}$ a $\mathrm{s} s$ e $\mathrm{r}$ e I I e s / $\mathrm{n}$ e $w$ s / a s s u r er-le-suivi-de-1\%E2\%80\%99int\%C3\%A9gration-r\%C3\%A9gionale-en -afrique

Dieye, C.T. (Page consultée le 16 janvier 2017). Que vaut l'Afrique dans le système commercial international ? Passerelles, Volume 16 - Number 8. 24 November 2015, disponible sur http://ww$w$. i c t s d. o r g / b r i d g e s - n e w s / passerelles/news/que-vaut-lafrique-dans-le-syst\%C3\%A8me-com mercial-international

Gbaguidi, Ochozias A. (Page consultée le 16 janvier 2017). Cinquante ans d'intégration régionale en Afrique : un bilan global, Épargne sans frontière « Techniques Financières et Développement 11, 2, $n^{\circ} 111$, disponible sur http://www.cairn.info/re$v \cup e-\dagger e c h n i q u e s-f i n a n c i e r e s-e \dagger d e v e l o p-$ pement-2013-2-page-47.htm.

Rouget, V. (Page consultée le 16 janvier 2017). Quel bilan pour l'intégration régionale en Afrique ? in http://terangaweb.com/quel-bilan-lintegration-regionale-en-afrique/

Union africaine (2015). Agenda 2063, l'Afrique que nous voulons, troisième édition, Addis Ababa, janvier, point 3.

Union africaine (Page consultée le 16 janvier 2017), Feuille de route pour une mise en place en 2017 d'une Continental Free Trade Area. http://www.au.int/en/ti/cfta/about

Vanheukelom, J. Byiers, B., Bilal, S. and Woolfrey, S. (2016). The Political Economy of Regional Integration in Africa. What drives and constrains regional organizations? Synthesis Report. Maastricht: ECDPM. 